\title{
Figure S2
}

\section{Promoter and coding regions of selected genes.}

G-box:

Gene: At $1 \mathrm{~g} 03630$, PORC

cacaaagtccactaccagttcataatttccacaccatcatcatcatgttaccaaacagtgatgcatcatatagaacatacgttcattatcattcattt tctatttatgtgttttggcttcattgattatcattttcatttcggtcataaattcaggttaatgtacaaaacataaaatcgaaatcaaactaagaaaat aacaaaagaaaaaaaatctattggaacaagagattgtaggcaggaagatcttgaatccgatgaatatcatccaaagaagetctctctccagctgattc cgagttgcttgtaatacttcctgataaacacacacagatatatacaattattaagaactaattctcaaaaaaaaaaaaaatgaaagagaaagttgata gattgagtgagaagagatacattgaattccatgtaagaagcgtgcattgcaagc cattgagcatccatcatcgcgaaagctatacagtatagtacatcga atgccctctcgtcttctgttaatcattggaatatgattcttgattatttagcgatcgtcatactacactactaacttttagaaagaaggttttattcagt ttaaagaacatattcctattgtttaccttctaagagtttgaggaaattcattcctggaagacattttggctttgctgcacaaaaacaaaacatgaatt taaagccttttagaatcgaaatcgtatgtgaaabaacaagtatgacctgaataaaatggtgacagtttaagaa acttacgattattttgtaaatcgagc aaccttcaca atttttatttgtat a a cacattatt tatttacgagtaatttaccggataagttcttgcagaaaatagtaaattttctagagcgataaatccgcaaccctacaaacaaatgtttaacaaaacag gcgtcaacactaagtatttttccttaagatcttttttgcaataagttaagtatagatcaaatcttacctgaaatcggtagatggattgggaccttgccat cccatttctttccattgttctgttactaaaccogtgagatgaacattcggaaacgcgacgttccacaatgctttaagtgattcctgccattaatcacgca aatatatttcacagaattcttatagctaaacaatatatctgcatgttgtttgctcactgcgttaaacatcttactgataaagtgaattcacctacctga tgatcaggacgttctcgtcgaaaggtacaaccatacgatcttgcagcctttgaagtctttcctcctatgtatgattgatttacacagatccaaaagtgt tacaaactatttataatgtct tgatttcattttatgaagatatatgaaacttgtgattatgctaacctgaagaggagacaaagtgtagtcgactgccttg tcttgacgtctattggagcgtgtgaagagccctcctatccatgatct tgttccaaccataccogtagctgtaaccactggttcactaatagaaaat tata ttcatatatgagataatagatctaaattatgtatctaataaactaatcacatgcatatttctatggtgtgtcatttataccctatgatatatctagcaat atatactcactaa a a a atatttagccaa at gagctattacgtgagcgga atatactcact (1) agaacaatg tatatgcaagattgcagtggtgtcgttgat tactctcaatttgatgataagattcaaatttgatatgagtttggagaagaagaagatgacg aagagatcattacttggcgatgagacgaa caagcaggaaagtattgcctacgcttcctgaacttcatgagatggacacaaatgttcaaagaatagaagat aactaatattgatatcttttaaactaagagaacaaagatgtcaaaaccgacataaatgcatggaaagaagaatttaggtataataatcctacatccaag ttatctagtcatcaaaacagagattagttaatgattttgcaaaagctaagacttctctgtagagaattggacagaaagaaagaggaggaagatttttata cagagctaagttgatcacagataagttttagtgttttatttggttgcgtacaggtcagagcttattaattttaaaatagaaaagcattgcgtgacta ttttaacacgtttaactaaactagacagaattgtgggttgttctgtataaattttgtatttgtatttttatttactcctttgagttaatttagagaatga caagaagatgatagaatcaaatgactaaatataaatgagctaaagttctaattcgatttctaaatattcaaaagttccatcatcatttccattttcact tagtcaattttggtataattgggctcaaaaggrttctctaaagttgtagctacataatagcgcaattaaggatgttttctttcataacttttat tagtcaaat gta (

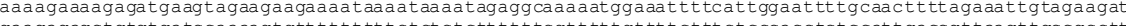
gaagagagctgtgtgatccaaaagtgtttttttt tctctctcttttttcgtttttgttttctttctccacctctccattgaccgttaagttgccgcctt cttctctcttccaccaaattgttcttgacacatttggacggtccagattcattacagttcacctatcaacggtgatcagatcagttgagagttaacaaaa caatattaagcccatctcaaggcccaataaacgagagatgaagaatctgaaactagtgat ctgattggttgagggaaaagctgtagcctggaaga cac gtgtcactaacccaaactaccttatcttctg tcgctcctcaattcggggaaatcgaagacactgtgacactctagcaccttcagttccg tacaaca

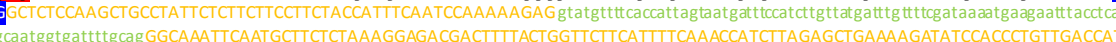
AAGgtaaagtcaatcaccctatacgatgtgaaattctcaaaaaaagatatcgaaaa cgtcctgagg tttatactctgtttcgttgcag GAACAGAGAAGACAGAAACCGCGATTITCCACCGGTATTC GTGCACAGACA GTTACAGCCACGCCGCCGGCAAACGAAGCATCGCCGGAGCAAAAGAAGACTGAGAGAAAAGGACTGCAGTGATCACTGGAGCTTCGTCT GGTTI GTGCACAGACAGTTACAGCCACGCCGCCGGCAAACGAAGCATCGCCGGAGCAAAAGAAGACTGAGAGAAAAGGCACTGCAGTGATCACTGGAGCTTCGTCTGGTT TCCAAGGAAGATTACACAGTGATGCATCTTGATCTTGCTTCGCTTGAAAGCGTGAAGCAATTCGTTGAAAATTTCCGGAGAACAGAACAACCACTCGATGTTCTTGTCT GCAATGCCGCG GTTTACCAACCAACT GCTAAAGAGCCTTCTTTTACAGCT GAAGGCTTTGAGATAAGCGTT GGAACCAACCATCT CG GTCACTTTCTTCTCTCAAGATT aatgaatgtt AACA GTTCAATGATAGATGGAGGAGAGTTGATGGAGCAAAGGCTTACAAAGACA GCAAAGTATGCAATATGCTGACAATGCA GGAGCTTCACAGACGTIACCACGA AACAGTTCAATGATAGATGGAGGAGAGTTTGATGGAGCAAAGGCTTACAAAG ACAGCAAAGTATGCAATATGCTGACAATGCAGGAGCTTCACAGACGTTACCACGA

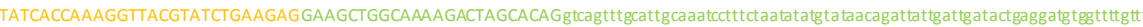
tagGTTGTGAGTGACCCTAGTCTTGGGA.GTCAGGAGTGTATTGGAGTTGGAACAAT

Gene: At5g17230, PSY

agtagccaagctaattatcactatctctaaacgcttgtgattctagattgtacacgttataagtaccaggatctatggaattaagttgagtaaaaacaaa gagtgatagtcactgactcaacaaaacgtcttggtcagtatatataacttactaaaaaacaattaacttgttataccatttgaaatttactactca tcaatcatcatgtctgttaaattttcagtatattataaaacgcatttccattaaatcaaagttaattatgccaatttcagtattctattgataactcg ggctaatccacatcgaagctcgaccaagtcccatgcatatggtctaggtgatcctctagaactagggcggttgacataaatttcgtacgaaaacaatact tttatcattcagtactaaaccaaaaaagcggtttctttacgagagaatgtatttgaaacaagatacggagaaaatacggaccagactaaaaataacaaa ggataaaaccaaaatcaagaagaaacttaaaagacaaaccttctgagcgcaagagagagggccaagttgcgaagacagtatgggacccaaataccgact aagcggaaatctaacttttaacttttcctaccaatgaacattga cacgtggaaaggcaacgtcgcccactttaccctccgcactttgaaatcttgata agct tatcgetctcccat tggccgtaggctc cacg tggcatctctctgatctctcttctgctaatccttttttacccaa actggcatgaccccacccta a accctattgtcctttctaccaacaattacttaatgacttatataatattctaattatattttaattatgtaattaaataactattgatcccatctcca tagtgacatgtctcacttttcetccacaacaaaatgcgtcactcttgattcatcatgttcgtagcaaaatccacgttattacaaaaaacgttat acatt ttgaaaggtaatccataaatagtgagatggatcaagttttctacgcaaccctcgtagaattttagcaatgatccaaagctactgtatatatatagttac gtttcgtaacaca catgtattttaccatgattatgcctctttttttttctttccacaacatatatgtatttacatccttatttggtttaggctgagtt

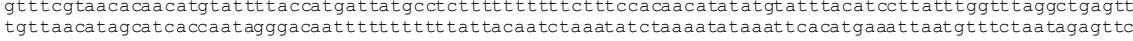
ttcaatcttcatgtacaattgcacatttttgtgaaattattattactttatcatttttctttaaaattaattcaccgagtaccgataaatgtttatatt gaatacgatttagtaacgacatatatgtttcgtcacatttttatcactatttgtaatatatctgttaatttgatgatgtagtttctgtttttgaattg aaaact tacaaagtttagaaaaagaatgtaaattagaa taaatacactttct gtatttttgaatactataataggctaaaatcacatcattttctaat gttgctttaataatatatatcaaataaggcttaaattacatttatataagaatattgtaatacaatatcaatttagagctattaatttacaattac aacctgttgcccaattgatgaataaatcaaacatgtaattaaccaaactttctaattgatcaggaatcaatttagctaaataataagacataaatttggc caagaaaagaagagaaa catgttaaa taggtaatgggttttaatattgggatagt tacctttatttgaaaactattaaggtttgatttaagacacgag tcactcgagagtcgcatctcgccagtcaaaactaaattaaaaactatatagacccaaaaataaaataacgacttttgtatttatactggcaagatt ctcattaaaacccagcaaaaaaaaaaaaaaagacaaagaaggaagaaa $t$ a tgagtgggt gagaattttttacgatagaggaagagacagcatcatcta cttgtgttgtctgtgtacatatattacagtaagcgttgcaatataacttcttgaggatcttctcacattaatgggtcaaaccttttgctcttccttttg ttaatttagtgtttgacaatctcctcctccttctccttct tcttcaaagttttgtcgcagtatctattgttcttacagagagaaaggtaggcttttgtgt ccatcactcattt cgctcgtttggttaagcttcatctgccatgtggttcactgttttgatacttttgggetcttatacctaatgttgatgtaacacaac
gatacataatctaatttgtttcgagtggaaaatagttgaacccagttgagtatttagccaatacgattagaggatttacttgtttttctctttcatg 
ttcttcatttgcttttgaagatcttcctctttatgtttgtgtctcttcgctattttacttgcttgaagaaattgagtttctcatctcgcttaaatttggc tttatatacagatttagagatctcgattcctaatctacttgtttttgtattcaatttgcaggaaagctttagtcttttaccagtttgatccaattctggg tttcactgaaaaaagttgggagtttgattcttctaactgtagaagaaacagag tcaacagaagaaactaaaaagttgagatttttctctcacgcgct

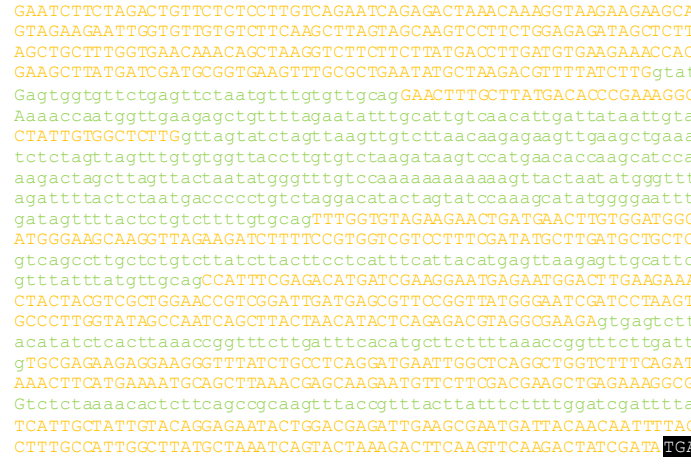

actattgttcccacacacaaaattattcttagtatttggtagctgtagctgttgtaatttgaagtccacttgataacttccttgtatttgtaggcgaag tatt tagaagctttagcctctgagacacttctcacaagcgatgaagcagtagttgtagcagcagcagctgaagcagtcgcccttgcaagagctgctgtca aagttgccaaaga tgcaacat tatt taagaacagtaaca acacgaactattaa cttcgtcaacggccoacaacgctccaagtgggaccagtt tactga gaaggaacgtgctggcatattggggcatctagcggtttcggacaatggaattgtgagtgataaaatcactgcatctgcctctaacaaagagtctattggt gatttagaatcagaaaacaagaagaagttgagcttctggaggagcaaccttcagtgagtttagctgtgagatctacacgtgaaactgaaaggaaagctc ggagggcaaaagggttagagaaaactgcatcaggtattccgtctgtgaagactggttcgagccctaaaaagaaacgtcttgttgcgcaggaagttgatca taatgatcctttgcgttatctaagaatgacaacaagcagttccaagcttctcactgtcagagaagaacatgagctgtcggcaggaatacaggtttgtcac caatattccaaattctaaactaaccacctccctttttctacatcgcgtctgagacatcaca aacactcaccettcgtaggactctagattctaggcgaaa atgatgtctacaaaatctttagatgtttgagaatgacgaaattagtggttagtggtagaatccaagatcgcttctttgtgctttctagccaatatatcat ctgaagttctgtgaaagctaattatcctgacatgcttgtcttaaattgaattgtcacctttttgttcttattatgtagccattcaccaactgtttagtg ttgatagaaa tgggaaacgtatgttcagctttggaagaatcaacat tacttgtgaacgttatttcttcttctctgtaatttttcttatctttctttatt gtggttttgatcacacactttggtttctttgtttaggaccttctgaagttagaaagacttcaaacagagcttacagagcgtagtggacgtcagccaacct ttgcgcagtgggcttctgctgctggagtcgatcagaaatcattaaggcaacgtatacatcatggcacactatgcaaagacaaaatgatcaaaagcaacat tcgactcgttatttcgattgcaaagaattatcaaggagctgggatgaacctccaagatcttgtccaggtttgagcttttctctccttatttttttttgta ataacacatctgtaagcagtagtgatgaaagtgatagatattgaacaccttgctcaatattttcgaatggtcacaggaagggtgcagagggcttg tga ggggagcagagaagtttgatgctacaaagggttt aaattt cgact tacgcgcattggtggatcaagcaagctgtgcggaagtctctctctgatcagtc

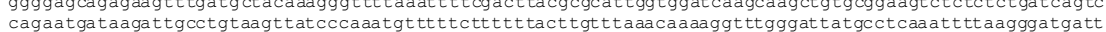
ggcagtttcacatggtggaagcaacatatagggtgaaagaggcacgaaagcaactgtacagtgaaaccggtaagc acccaagaacgaagaaattgcaga ggcagttcacatgg ggaagcaacatatagggtgaaagaggcacgaaagcaactgtacagtgaaaccggtaagcacccaagaacgaagaaattgcaga grtacagggctg cratgaagagactcatggcggttctactctctcctaaacctccgaggtcgctagaccagaaaatcggaatgaatcaaacctcaaa ccttcggttggtaccacctgacacaacacacaccataca aca tagacttgttgtttgtctaaaa tagtaaataatatgagatgggtttg taatgtaaaa gggtacaagggagaaacaagtgatacgt tggagatttgggatggaggatgggagaatgaagacgttgcaagagataggagagatgatgggagtgagcagg gagagagtaagacagatagagtcatctgcattcaggaactaaagaacaagaagagaaacaaccatttgcagcaatacttgg ttgcacaatcataattct ttttctct tcaatatggaatgttttgtgcataga acccacccacctgagcataacaagcagtaa ttattactttttagcatttacgattcttgtgacaa ctactaaatcgaaaaaattattttaaaactaaaagaaattgttgtctgaaaaacataagtgatatacaacaataaataaatatctcttataagaatttg ggaaaaaaaaatccaaagattagagatatttgggtggagaaaacaaccgcctaggacaaaaaaaattgtgggtagaggaggaggaggaggctttgttc acacaccacacgacggaacattctcttccgagtcttatccaaagcaaatcatatttgcttaaatatcttcttctcoggctctgtctctgtctctct tc ttcccgcggagct t tcagtttttgtttggtaacatcgtttctctttctcactaa ctctgatatcatattatcaatttttccttttcaacacccaaaatca caatctttcagctttattctgttttccttgcttgagaattgtgttcgattcatgtctacgaactgaaatcacaaattttgccaacgttttatgattcct ggttccgtattttttttttgtaatcaatagttagctcatttcgaataatcaaactcaattttattgttcatgatttgttatctgggataaaagagagta atgtgctaattatgtatactcaggtattgcttggtgtggggaagattagatagtgtgaag

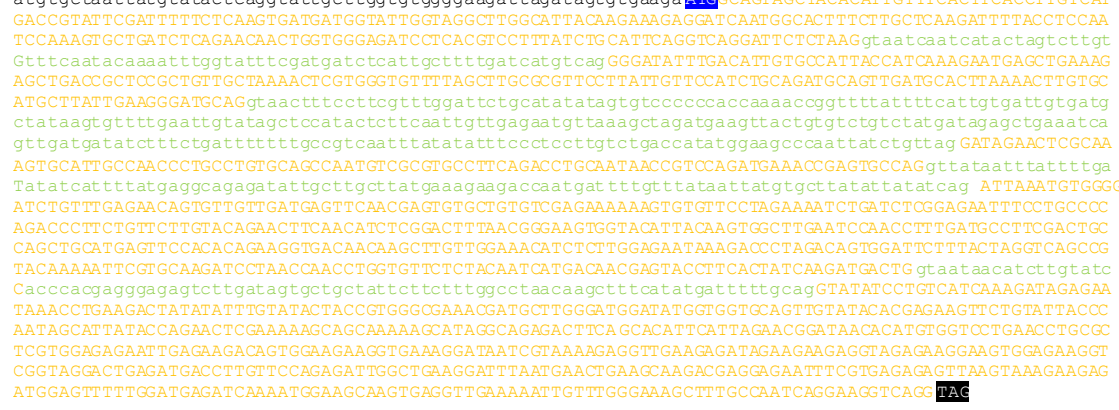

TG: transcription start site

GA, TAA: transctiption stop site

G-box :

Gene: At5g13630 GUN5

aacatatttaagccttaagtaatgaagaaagaaacgtaacatggaacagaataatagaaagaacgatgagaaaagaacctctgtgttctgaaacgaaagc cgccagtcatatgcagccatgtacatattttctcttcatatccaatatgtgcaaggttagcaatcagcactgcccagacaaagtagccaggagcaaagt agtcagcagccacgagtcctgatacagctcgaactctaataccagctggatccaaccagtttcattgtcaagtgacatgtgttccacccaacatagagg cctgaatcaaagagataattgattagttaagatactatcaactataccactgttaccatcaacaataacattgcccatctctaccatagcaaaa acatga acactgagccgaa agaagaagatgcagatcgtaacaaagatgcaacccacaaaa cactttttttttaatatatccatagcagggtcgggaggagttcaaa gctggataacaga acgaggatggacgaaat tcaagacatgtgaataaactcgatg tgaaactttgaaattgagaaaaaattgagaaaagcacattgagc tgaagatcaaaaggaactagctttaattacacatatacatagttgaataataaagcaacagtcctctatcacagcctgaacacactttccacagatgt

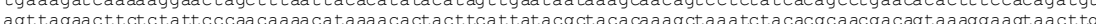
ttatgacg agaatagtccaagccaattacgtccttggaacgcttaccttacacatacagaaatagtctcttaagaaatagcgtttcacatcaaaaaaaaaaag 
taaagagtaagacagacaaacatggaat taaagt t tgagtgatgttgaagaagcaacaagtgcgtgatctcatcagatccaatccaaatcccaa tataaa ggaagagtgagaattgttgagctcaccttt tg tagacttcaccaaagttccaccccacaaacgttttctaaataaaccatcagcgcattgtttgccttc ccaaagctcgagcccaccggtgacaatcccaggaatgaagacaacaggatgtttcgccttaagacct tcttttttgagct taacaccgggcgggtcaggc aa aggacccgtgattcgctccgttacatactgagggaagctcgcaggcattgcgttgtaaaggaagagaagaaaccaccaggttacaca cacacacccaa tgaaccaacaacaagaatcgatgcacgaccacttccctcctccgttcgatttct tatggtgggatttggaagattcgtgtggtttcttttgcgaatcctc atcgtgcaccacctcttcagatggcggcgtcgatggtttctccgtcggctttttccgatgaataaggggcatatctgtcact tgaaattaaaccaggtta agctatacggact totcaaatctcgtccaggaatttagaacacagattagggattgttagatcagtcgagagagacaaagagctgtttctctgcgaatt tctggaaccaatcaaatcaaatt tgtctcttt tgtttattgtcgacttt ctgttttttctgttctggtattattcctccaagggactcggaccggatg gcaaact tgaccactttgctcctggttcaggttgcggttcatggtcaaccttagacccgacctatccgtttcgttgatttcattaattagtctgcgttt tcttgggtggagtgcagacagatt t tct t ctgcagtaggcgttaatccgataggctcttgt tgggcctcaaatagt tcagtaacttttcgtggggct tt tagtatagcccatgtattaataacaatat tacacttgggaaatgacaaaaaaaaaagagaatgaagcggtactcttatatcaaaaaacatattgtat atcttcgaatatgatgcaactcatgcaacaacaatactagtaggctacatg tattgctccatttaattacatataacttaatattctaattcataaactt tcttttaattatatattttatgtaagttttaaatattgaaaaaaaaaagttttaaatattgaacatgataagtttttacatccatttattataaaa aatagtgatgatgatgatcaaattttgagtacattttagagtgttttaacttttacatttgttgttacaattgttatgagtctgatatttcaaatttc gttttcaaagaaaatcctgagtttaccttaaaagattaaagccagtttttaagaaataagtagaaaa tatcaaaccagacctagctgaaccaaaatt at acaaaatctagaccggat taa accggctacccgacgaatatgcctagtaaaagtgtcgtcatcggaaaagaatcttttacatggctgaaaccgcaaat acgatcttcaagagagcaacataaaaatacttgtcaaataagtaaagaaaataagacactcaatatccacactcccaaccatgaaaatatgaatagaaa tctcaatgagccaatcagaggccagcaaagtccatctcattgtcccaagcggatatacatccgacaaaactcaacctcacaagtagactctcccttcttc cgtaactattcgtc cacai gtccttccetctcaccacttaccttcaaaccaacgcttctttttagttccttggtccgaagcgtttaccgatgagagaa tcataaactcccacttggagctcaaaagtgtaagagacaaccaacaaaaacgattcatctcttctcctatcct ctcctcttcgaattcaacgtttgga gaatccagcagccgcaaas

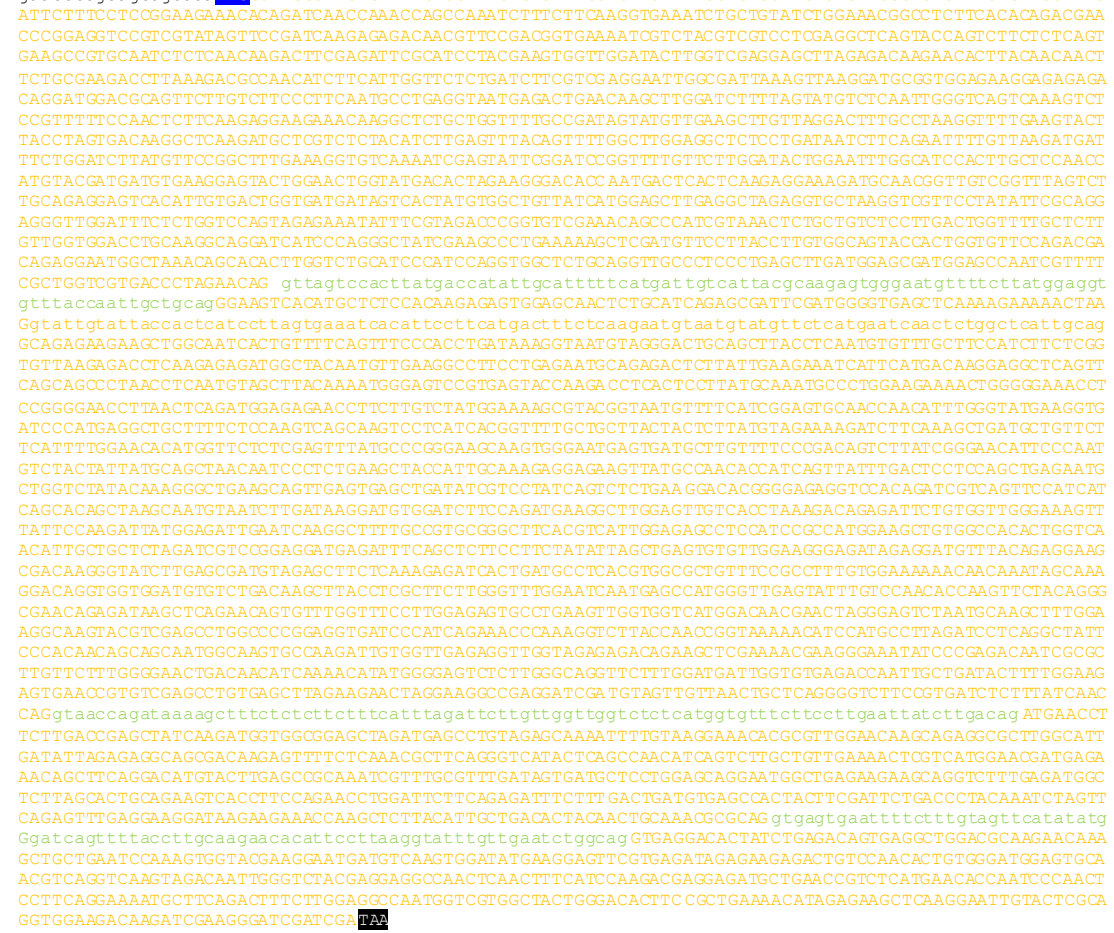

TG: transcription start site

-box:

Gene: At3g47470, LHCA4

ttccgaggttttcaaatcattcaactataataataaatgttttttatttgaatcaaaaactgatcatgggtttgaaactgaactaccacgaagagtctca atatttctaca tatcaaa tattccaaagggtacttgggtcggtgaaagcaacaaga taataaatttaaaggaaaacgttacaaggaaattgcatggaaat ttccatgaattggaatccaagacaatgaaagatgctctcactcaattttcaact ctattgtcttgtgttcttggatatgatgagaagttgattacattta ttattcaactatcaaatatatataaacatttgttttttcttatatgacaagattaacttccatgtctccatttggttcagtccaaaatttcaatttg atttgagatatatatatatatatatatata tatatatatatatatatatatactccacctattacttgaaagccaacatttcaagaacatat attaattttgaaatgaaa tacagattaattattaataacaagcattaagtttttattagtatgatcatcattaatgaaagcttgagtcgatggtagttt attaatttgaaatgaaatacagattaattattaataacaagcattaagttttattagtatgatcatcattaatgaaagcttgagtcgatggtagttt cttaggtttctcggtaggtataaagtatgaatataatacttcaattgaattgcggtgagtttaaagtagcattettcttccttggtaagtttct caacaattlacatggagactctetccgcagacttegtgagtaagatatgtttggtctecgtatacttaactaggaattataaalaggaccaactcaac ggattcgtctcttcttagcccaatctctctcctcaccattgtcatcttcttattcattctcaatctcatgatgataattcaagacttctctcttcttt ggattcgtctcttcttagcccaatctctctcctcaccattgtcatctccttattcattctcaatctcatgatgataattcaagacttctcttcttctttt ccctttcgtttccatctcttcttctctaatgcctacattctcttcacctcaataagaaacaataagcaaaataccgagctcccaatcataaagaaagt $t g$
ttgtcccaaatcgagcagatatcaaaacgtccgtggaagaagtcaaggcgataatcgatgactcggaagctt tatacgaatgtt tgattgaagaaggaga ttgtcccaaatcgagcaga tatcaaaacg tccgtggaagaagtcaaggcgataatcgatgactcggaagctttatacgaatgtttgattgaagaaggaga agagtact tgcttgagaagaatgaga tga tgggtaaggaga tagt laaagaggcgttlaggtta tlgatgagaa tcaggatgggtt ta tagatgaaaat ttgatttttatgagtttgtgaagcttatagagaagagcttttcgtgaagtagattggaataataataatagattgatatattcatgcttctctgaatt ctaatatatttct tcttctttagtgggtcgtcctctaatgaactttattttcctctgacaaact tatteacgttgatatattcatgcttctctgaatte caagaaagtgataata ta tgacaacgtaact taaatttgtgatgaatta gaagggaaaaagtatggaccataatcgaacaaataacttaattetcactataatataaagtgatcatacataatgtttatatagagcttgagactgc tacatctagctagtttcctaatacactttttcaataataaaagagaagagattaatttgtttttttttaaaaaggtcaagataatagtcaatctcatc atttgctccaaaattttagtgttaatcaaa tgtgggattatataacagactacaatgtaaatagtaggattctacaaactcaaaatatttgtaacagtt ttttct tttaa a tgtaat t cctact ( aggtaatctcaaatgtcactg taactctgtaagattttgaaagatgaacatgatcttgaaaagagagattaaagaaaagatggatttaagttcttcaa cactetcttectetettctectgggectaaacaacceaatatctcactettcaaacga gecegagattctcaaccttgacaagcccaaagtgaagat catatgccaattcagttccatttgattggttgacgagaatcctcactccactctaatattttaatccatt taattga cacgtgtaacaacaatggacc 
ctcctccgtcaaatcaaccacgtcatctaatccaacggtagatactcagtcatcaaaccacagccacaaaactcgtttcaagaaacgaataaacttacaa aatcccctccaagtcttatttcttcacaaccatctctgaactcatctttcaatctctcttgattccaatccattcttcttcaagtgccgggttctcgggt

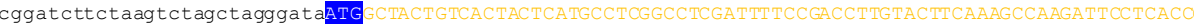

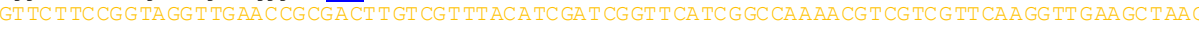

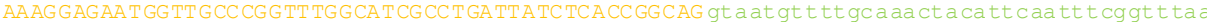

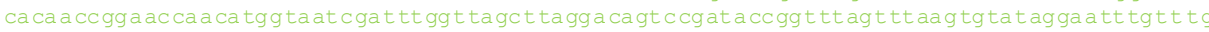
aacacaaccg tgactaa tcagt ttggattggttaggttagaccggacttggttga taccggtt tagt taa taa tg gtcactcactc actct ta tacatat tgcag TCTTGCCGGTGACAAT GG TTTGACCCGTTGGGTCTAGCAGAGGATCCA GA GAACTTGAAA TGGTTC GTCCA GGCA GAGCT GGTCAACG GACGAT GG GCTAT GCTCG GT GTCGCT GG GAT GCTTT TGCC G GAAGTTTTCACCAA GAT CG g tga

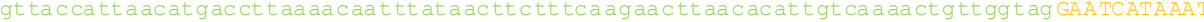
GTTC TGAGT GGTA C GA TGCTGGGAAA GAGCA GTATTTTGCATCGTCGTCGACATTGTTCGTGATCGA GT TCATA TTGTTTCA TT A CGTT GAGATCAGACGGT GGCAA GACATCAA GAACCCA GAAGT GT GAACCAAGACCCTATCT TTAAGCAA TACAGCTTAC CTAAG GT GAA GT TG GT TCCCT GGT GGAATCT T TAACCCGCT TAACT T TGCTCCT AC GCAAGA GGCCAAG GA GAAAGA GC TA GCAAACG gt

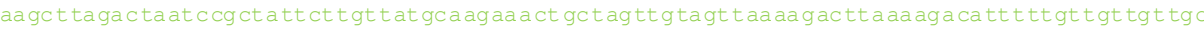
agGGA GGTT GGCGAT GT TGGCATTCTTA GGGTTTGTGGTTCAACACAATGTGACT GGAAAAGGACCATTT GA GAATCT GT TGCAGC ACTT TCTGACCCATGGCACAACACTATTGTCCAAACCTTCAACTAA

\section{Figure S2}

